this line of advance was the invention of the Raper autoleveller, a drawing machine which maintains thickness regularity by automatically taking account of the irregularity of the ingoing material. Mr. George Raper brought his idea to Torridon for early development, and after further development in a mill it was put into commercial production. This system of drawing, in conjunction with the Ambler 'superdraft' system of spinning (developed by Mr. Geoffrey Ambler with the co-operation of the University of Leeds), is now accspted as one of the modern methods of worsted drawing and spinning.

A more recent achievement, used as a part of a shortened drawing system, is the Association's rotating can system, in which twist is inserted by means of a rotating can instead of a spindle. Other investigations in improved methods of worsted drawing are being pursued at Torridon, including the use of closed loop servo-techniques for autolevelling.

An important aspect of the irregularity of the ultimate yarn is its effect on the appearance of the fabric. The Association has pioneered the expression of this irregularity in numerical form, and the problem of linking numerical assessment with visual judgment is still being studied.

Many other projects are in hand, both in scouring and combing and in drawing and spinning. Members' day-to-day problems often lead to some profitable line of research the solution of which benefits the industry as a whole. The use of man-made fibres in blends with wool raises difficulties at various stages of processing. New types of machinery introduce new aspects of old ones. This kind of service to the industry will be greatly facilitated by the increase of machinery and laboratory space in the new Worsted Block.

H. LEMON

\title{
APPLICATIONS OF INTERFERENCE MICROSCOPY
}

\begin{abstract}
A SYMPOSIUM on this subject was held at the Royal Microscopical Society's London headquarters on March 15. The president, Dr. V. E. Cosslett, was in the chair and the audience included a number of visitors from abroad. It is nearly ten years since the first interference microscopes suitable for general use began to appear, and the purpose of the meeting was to take stock of the present position and to review some of the main applications of the instrument in various fields.
\end{abstract}

In his introductory survey Dr. R. Barer (Oxford) dealt with the instrument, theory of operation and the principles underlying its use for 'weighing' cells. He paid tribute to the work of J. Dyson, F. H. Smith and J. St. L. Philpot, who made the development of modern high-aperture instruments possible, and discussed some of the problems common to all types of interference microscope. The operation of a twobeam interference microscope can be explained by means of a simple vector theory which shows how the optical path difference produced by a transparent specimen can be measured and also demonstrates the similarity between interference and phase-contrast microscopy. The latter can be regarded as a form of interference microscopy in which the two interfering beams are imperfectly separated. This causes the edge of a refractile object to be surrounded by a halo and the contrast of edges and discontinuities is accentuated. These apparent defects of phase contrast are not necessarily a disadvantage for purely observational worle; and the image of a cell seen through an interference microscope is often disappointingly 'flat' and lacking in internal contrast as compared with a phase-contrast image. Dr. Barer concluded by giving a practical interpretation of the meaning of optical path difference, the quantity normally measured with an interference microscope. The refractive index of a solution varies linearly with its concentration and since the optical path difference is defined as the product of the object thickness and the difference in refractive index between the object and the surrounding medium, it follows that the optical path difference is proportional to the dry mass per unit area. This is an important quantity in cytology, but present methods are mainly adapted to point-by-point measurements and the integration of dry mass over the area of a cell might be very tedious. The full value of interference microscopy in biology will not be realized until automatic integrating devices, based on rapid electronic scanning and capable of 'weighing' a cell in a fraction of a second, have been developed. This is a technical problem and by no means insoluble.

Dr. K. F. A. Ross (Leyden) described how interference microscopy can be used to measure the mass or solid concentration of cell inclusions. It is necessary to overcome the difficulty that the inclusion is surrounded by a layer of cytoplasm of unknown thickness. One method is to immerse the cell in an isotonio protein solution having the same refractive index as the cytoplasm. The difference in refractive index or concentration between the inclusion and the cytoplasm can then be found. Measurements of this type have been made on the Nebenkern of the locust spermatid, a body formed by the fusion of mitochondria. Values in the range of 21-26 per cent for solid concentration were obtained. Work on lipid droplets in various cells has thrown some light on their physical state. The refractive index of droplets known to contain mainly phospholipids is considerably lower (about 1.42 ) than that of any pure lipid substance. This suggests that in their natural state these bodies are highly hydrated, a conclusion supported by evidence from polarization and electron microscopy. Finally, Dr. Ross described measurements on different regions of the giant salivary gland chromosomes of Chironomus. A number of different methods all showed that the euchromatic regions contained a higher concentration of solids (20-25 per cent) than the heterochromatic regions (10-15 per cent).

Dr. E. J. Ambrose (London) described his work on cell division. The instrument used was a polarizing type of interference microscope with a birefringent wedge mounted in the eyepieco. Time-lapse cinéfilms in colour were taken of dividing cells, simultaneously with a coloured image of the wedge. The optical path difference of any part of the cell could 
be measured by matching its colour against the scale of colours exhibited by the wedge. In some cases it was necessary to compress the cells in order to obtain good optical conditions, but with plant endosperm the cells could be mounted on a thin film of agar on which they were made to spread out by surface tension. Measurements were made on the cytoplasm, nuclear sap, chromosomes, spindle and cell plate. A good, correlation was found between regions of high mass per unit area and the development of birefringence. Dr. Ambrose concluded by showing some excellent colour films of cell division.

The three papers in the afternoon session were devoted to non-biological applications. Dr. S. Goldstaub (Strasbourg) first described his work on crystal growth. The interference fringes surrounding a crystal immersed in its mother liquor are in fact lines of equal concentration. In regular growth these fringes tend to cut across the corners of the crystal, so that the crystal surface is not a region of constant concentration. Nevertheless, since the fringes move parallel to themselves during growth, an equal mass of material is deposited over the whole surface. In dendritic growth, on the other hand, the fringes do not cut the crystal surface. Dr. Goldstaub also gave an interesting account of the use of the interference microscope for studying the behaviour of salt solutions surrounding isolated particles of ion exchange resins. The flow of ions from the solution to the resin gives rise to concentration gradients, and hence to fringes around the particles so that the exchange process can be observed directly. A colour film illustrating some of these points was shown.

Dr. R. C. Faust (Imperial Chemical Industries, Ltd., Northwich) discussed the study of optically heterogeneous specimens with special reference to textile fibres. Many fibres are far from homogeneous and some possess a surface layer or 'skin' that differs markedly from the central core. A knowledge of the absolute refractive indices at different parts of a fibre is an important guide to the degree of molecular packing and orientation. A convenient method of observing the variation in refractive index is to immerse the fibre in a liquid of known refractive index and to study the way in which interference fringes are deviated in traversing the image of the fibre. At those points at which the fringe is undeviated the refractive index of the fibre matches that of the liquid. If the refractive index of the liquid is changed by varying the wave-length or the temperature, new match positions are obtained. A map of refractive index variation can thus be built up. In some unstretched fibres the refractive index for polarized light vibrating parallel to the axis remains essentially constant across the fibre whereas for light vibrating perpendicular to the axis the index falls rapidly from the edge to the centre, so that the birefringence is maximal at the edge. After stretching, however, both refractive indices vary together, giving uniform birefringence. Dr. Faust emphasized that the optical properties of fibres vary considerably, depending on the method of manufacture.

A glimpse into the future was provided by the last speaker, M. Marcel Locquin (Paris). He described how microscopes are needed to magnify the very small 'memories' used in machines for automatic documentation and translation. The 'memories' are produced as photomechanical negatives by a process similar to that used for making graticules. Although in most European languages 40,000 phrases are in common use, about 90 per cent of a language can be translated with only $2,000-4,000$ phrases. A common word can be represented by one or more letters using speedwriting shorthand. Additional letters are used to represent the dependence of one word on another. The encoded phrases are made into a memory which can be a thousand times smaller than a magnetic type of memory. The memory negative is magnified through a microscope and its enlarged image falls on to a 'question' in the form of a photographic positive. A photoelectric system measures the amount of light transmitted by the overlapping images; if the memory and the question are identical no light is transmitted and various degrees of similarity can be selected according to the light transmitted. Such a system can be used for selecting papers on similar subjects without human intervention. The particular advantages of an interference microscope for this work are that it provides a means of contrast variation and that silver grains in the photographic emulsion are rendered essentially transparent by interference contrast, so that the image is effectively grain-free. However, M. Locquin felt that there were possibilities for reducing the size of the memories still further and looked forward to the day when they would have to be magnified by means of an electron microscope.
R. BARER

\section{THE CARNEGIE TRUST FOR THE UNIVERSITIES OF SCOTLAND}

$\mathrm{T}$ HE fifty-ninth annual report of the Carnegie Trust for the Universities of Scotland, covering the year 1959-60, includes that of the Executive Committee, the abstract of financial accounts for the year ended September 30, 1960, lists of publications during the year from Fellows, Scholars and recipients of grants, and of papers published by the Royal Society of Edinburgh assisted by Carnegie grants, and the usual reports on the work of Fellows and Scholars and on research grants and travel grants awarded during the year*.

* The Carnegie Trust for the Universities of Scotland. Fifty-ninth Annual Report for the year 1959-60 submitted by the Executive Committee to the Trustees on 27th February, 1961. Pp. iv +66 . (Edinburgh: Carnegie Trust for the Universities of Scotland, 1961.)
In annual and non-recurrent grants in the 1957-62 quinquennial distribution the heaviest items have been more than $£ 18,000$ by St. Andrews on the new Biology building, a similar sum by Glasgow on the new Arts building, and more than $£ 23,000$ on the new Physical Education Centre; more than $£ 26,000$ by Aberdeen on the new hall of residence, Crombie Hall, and nearly $£ 9,000$ by Edinburgh on the new hall of residence at Salisbury Greon (Holland House). The Committee is now considering its policy with regard to capital grants for the period 1962-67, and has agreed to ask each university, in formulating proposals for use of the Trust's funds in this eleventh quinquennial distribution, to concentrate on projects 